\title{
Exploratory Studies Detecting Secondary Structures in Medium Resolution 3D Cryo-EM Images Using Deep Convolutional Neural Networks
}

\author{
Devin Haslam \\ Department of Computer Science \\ Old Dominion University \\ Norfolk, VA, 23529 \\ dhas1002@odu.edu
}

\author{
Tao Zeng \\ Department of Computer Science \\ Washington State University \\ Pullman, WA 99164 \\ tao.zeng@wsu.edu \\ Jing $\mathrm{He}^{\dagger}$ \\ Department of Computer Science \\ Old Dominion University \\ Norfolk, VA, 23529 \\ jhe@cs.odu.edu
}

\author{
Rongjian Li \\ KeyBank \\ Cleveland, $\mathrm{OH} 44114$ \\ rli@cs.odu.edu
}

\begin{abstract}
Cryo-electron microscopy (cryo-EM) is an emerging biophysical technique for structural determination of protein complexes. However, accurate detection of secondary structures is still challenging when cryo-EM density maps are at medium resolutions (5-10 $\AA$ ). Most of existing methods are image processing methods that do not fully utilize available images in the cryo-EM database. In this paper, we present a deep learning approach to segment secondary structure elements as helices and $\beta$-sheets from medium-resolution density maps. The proposed 3D convolutional neural network is shown to detect secondary structure locations with an F1 score between 0.79 and 0.88 for six simulated test cases. The architecture was also applied to an experimentally-derived cryo-EM density map with good accuracy.
\end{abstract}

\section{CCS CONCEPTS}

- Computer vision $\rightarrow$ Computer vision problems $\rightarrow$ Image segmentation;

- Applied computing $\rightarrow$ Life and medical sciences $\rightarrow$

Computational biology $\rightarrow$ Imaging

\section{KEYWORDS}

Deep Learning, Neural Networks, Fully Convolutional, Protein, Secondary Structure, Cryo-electron Microscopy

$\dagger$ Corresponding author

Permission to make digital or hard copies of all or part of this work for personal or classroom use is granted without fee provided that copies are not made or distributed for profit or commercial advantage and that copies bear this notice and the full citation on the first page. Copyrights for components of this work owned by others than ACM must be honored. Abstracting with credit is permitted. To copy otherwise, or republish, to post on servers or to redistribute to lists, requires prior specific permission and/or a fee. Request permissions from Permissions@acm.org. ACM-BCB'18, August 29-September 1, 2018, Washington, DC, USA

(C) 2018 Association for Computing Machinery.

ACM ISBN 978-1-4503-5794-4/18/08 _\$15.00

https://doi.org/10.1145/3233547.3233709

\section{INTRODUCTION}

Proteins are imperative to living cells. The threedimensional (3D) structure of a protein determines the function of the protein. Cryo-electron microscopy (cryo-EM) is an important technique in molecular structure determination. Using cryo-EM, a growing number of large molecular complexes have been resolved to atomic resolutions $[1,2]$. However, for cryo-EM density maps with a medium resolution (5-10 $\AA$ ), it is much more challenging to recognize detailed molecular features. In most cases, it is not possible to derive atomic structures from these medium resolution images without the knowledge of known atomic structures as templates. When a template structure is available, fitting is used to derive atomic structure $[3,5]$. When no suitable template structures are available, matching secondary structures that are detected from the 3D image and those predicted from the sequence of the protein may suggest possible topologies of secondary structures [6-10].

The most common secondary structure elements (SSEs) in a medium-resolution density map are $\alpha$-helices and $\beta$-sheets. The major difficulty of detecting secondary structures in such density maps is that the patterns of the SSEs can be indistinguishable from their narrowly located neighbors. Many methods have been developed to detect SSEs at medium resolutions. These approaches are mostly based on image-processing techniques. A helix is often identified using cylinder-like templates or carefully-designed cylinder-like features. A $\beta$-sheet is identified using plane-like templates or features. The drawbacks of these methods include carefully selected parameters and under-utilizing large amount of existing density maps in the database. If SSEs could be more accurately detected, this would be an important step to automatically resolve protein structures from cryo-EM images at medium resolutions [11-17].

Generally, long $\alpha$-helices, such as those with more than 20 amino acids, can be detected by a variety of methods. On the other hand, short $\alpha$-helices can be easily confused with turns/loops. Similarly, large $\beta$-sheets show unique characteristics while small $\beta$ - 
sheets might be confused with an $\alpha$-helix. Due to the small spacing of $\beta$-strands at about $4.5 \AA$, these strands are often not visible in a medium-resolution density map. Several methods have been proposed to predict traces of $\beta$ - strands from segmented $\beta$-sheet regions $[18,19]$. As machine learning methods continue to show their merit in image processing tasks, several approaches have been taken to solve the problem presented. The authors of [20] used nested $\mathrm{K}$ nearest neighbors classifiers to detect $\alpha$-helices. In addition, methods using support vector machines (SVM) have also been employed to identify $\alpha$ - helices and $\beta$-sheets [21]. However, empirically-derived features may not be representative enough to obtain state of the art accuracy. Most recently, Li et al. has shown potential of convolutional neural networks (CNNs) achieving good performance [22].

Convolutional neural networks utilize arranged layers to learn complex features. CNNs have been shown to produce state of the art performance in a variety of image related applications [2328]. More recently, CNNs have been extended to tasks involving image segmentation with good accuracy [29-31]. CNNs are appealing due to their ability to learn features with trainable parameters in tasks that require nonlinear relationships. Due to these advantages, we explore $\mathrm{CNNs}$ to segment secondary structures from cryo-EM 3D density maps.

\section{METHODS}

\subsection{Architecture and Parameters}

Several challenges are presented when attempting to segment secondary structures from a cryo-EM density map. One of these challenges is the large diversity of proteins in the database. The architecture used to segment these SSEs must be able to learn features from multiple scales. A second challenge existing is the varying sizes of proteins with in the database. In order to overcome this problem, we train and test with patches of size $48 \times 48 \times 48$. A visualization of the patch can be seen in Figure 1. We attempted to find a size that would be small enough to eliminate the need for padding the 3D images, while still being large enough to hold important information when the receptive field is reduced to its smallest window.

Inspired by 3D-UNET [32], we implemented a similar model. This model consists of an analysis path and a synthesis path. In the analysis path, each layer consists of two $3 \times 3 \times 3$ convolutions, both followed by a batch normalization and a relu operation. Each layer in the analysis path is ended by a $3 \times 3 \times 3$ max pool with a stride of two. By using a stride of two, we reduced the receptive field by a factor of two at the end of each layer in this path. After three layers that use increasingly more features, the analysis path has ended. The receptive field at the end of the analysis path is now eight times smaller than the original input. The synthesis path is very similar except each layer is ended with a transposed convolution increasing the receptive field by a factor of two. We also concatenate the results of each layer in the analysis path with the results of each synthesis layer. In the last layer we use a $1 \times 1 \times 1$ convolution to decrease the amount of output channels to three labels. A more detailed description of the architecture can be seen in Figure 2.

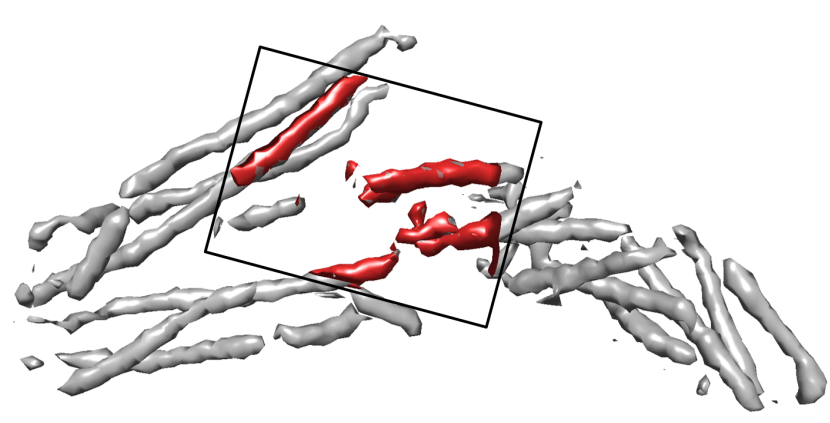

Figure 1. An example of the patch size in training and testing. The density within a patch (red) is superimposed on the entire $3 \mathrm{D}$ image simulated using the atomic structure of protein 2XS1 (PDB ID).

Small batches of four images and a dropout rate of $50 \%$ were used during training. Unlike the previous work using a CNN architecture [22], no post-processing was performed, yet the model produces equivalent results as those using postprocessing in the previous $\mathrm{CNN}$ architecture. Naturally, we used softmax with cross entropy to measure loss. In order to optimize this loss function, we employ an Adam optimizer with a 1e-4 training rate.

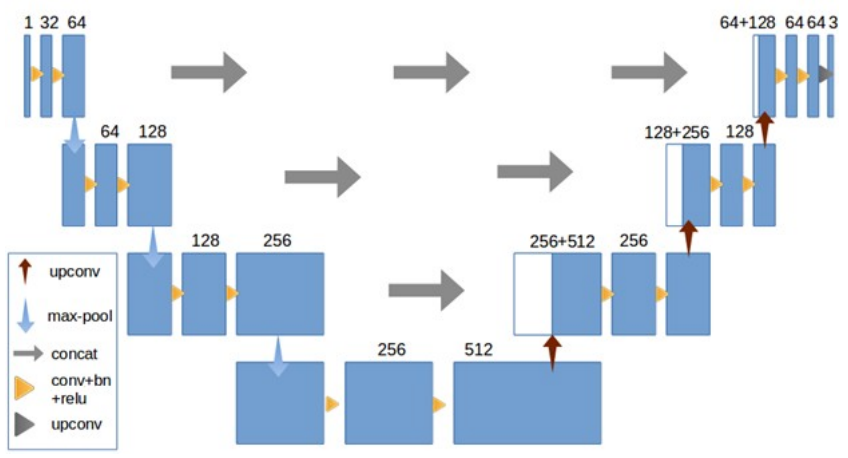

Figure 2. The 3D U-net architecture.

\subsection{Data}

We have used the presented architecture to test six simulated 3D images and one experimentally-derived cryo-EM density map. After collecting 31 atomic protein structures from the Protein Data Bank (PDB), we simulated each to $9 \AA$ resolution with a $1 \AA$ voxel size using UCSF Chimera [33]. Among the 313D images, 25 images were used for training, and the remaining six were used for testing. In order to fully utilize the simulated 3D images, each image was rotated around the $\mathrm{X}, \mathrm{Y}$, and $\mathrm{Z}$ axes with a random angle to produce $353 \mathrm{D}$ images as additional samples. Conversely, when using experimental data, we have downloaded each cryo-EM density map from Electron Microscopy Data Bank (EMDB) and the corresponding atomic structures from the PDB. Although there is a large number of cryo-EM maps with annotated resolution between $5-10 \AA$, only those with visually good quality were used for training. When evaluating our model 
on experimental data, we used 42 cryo-EM maps with a total of 67 chains for training. Much of the training data is unique, but there are a few chains in the set that are similar. The experimental data used for training and testing have voxel sizes between $0.82 \AA$ /voxel and $1.86 \AA$ /voxel. We expect the network to learn the characteristics of SSEs even when the voxel size might be different.

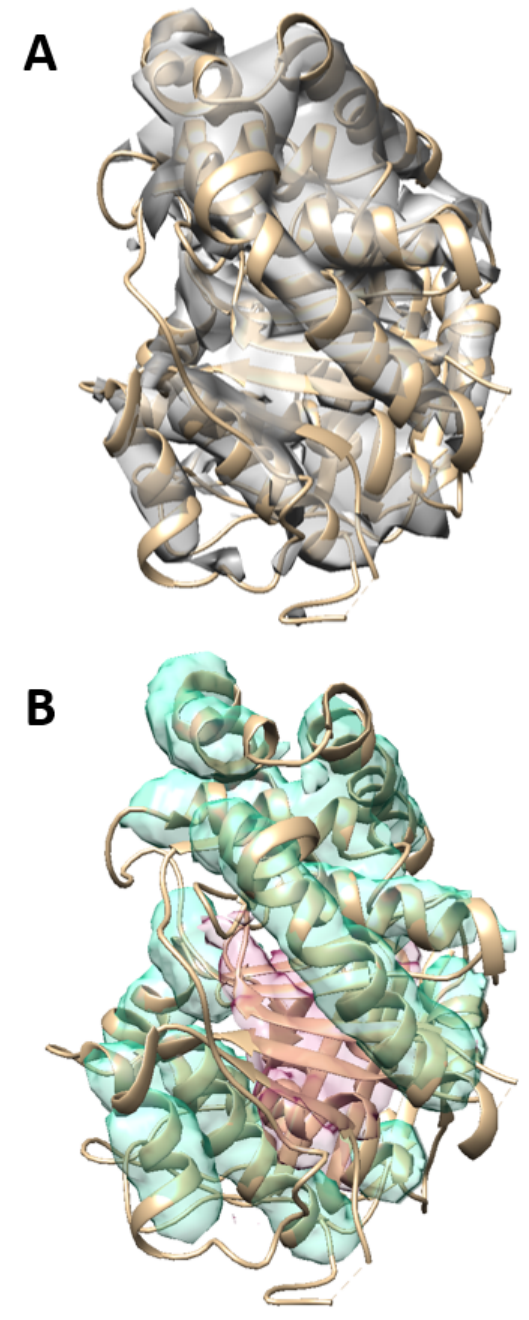

Figure 3. An example of secondary structure segmentation using the CNN architecture. (A) A 3D image simulated using the atomic structure of protein $3 j 7 i \_a$ (PDB ID) (shown in ribbon). (B) The detected helix regions (cyan), and $\beta$-sheet regions (pink) are superimposed with the atomic structure (ribbon).

\section{RESULTS}

An example of secondary structures segmented from a simulated 3D image is shown in Figure 3. This protein 3j7i_a (PDB
ID) has 17 helices and nine $\beta$-strands (Table 1). Visual inspection shows that both the helix regions and the $\beta$-sheet regions were identified correctly using the proposed $\mathrm{CNN}$ architecture. When testing, we also use patches of $48 \times 48 \times 48$. As an example for 3j7i_a, nine patches of $48 \times 48 \times 48$ were randomly selected from the entire density map. The accuracy of detected helix voxels was quantified for each patch using the F1 score. We observed that the F1 scores of different patches in a protein are similar. The averaged F1 score of nine patches in 3j7i_a is 0.806 for helix detection (Table 1). The average F1 score of helix, $\beta$-sheet, and background is 0.789 for all nine patches in protein 3j7i_a. The F1 scores for helix detection are between 0.734 and 0.872 for the six test cases (Table 1). The F1 scores for $\beta$-sheet detection are from 0.749 to 0.999 . The three cases with the highest F1 scores of $\beta$-sheets have small $\beta$-sheets with two strands only. The overall 3-class average of F1 scores are between 0.795 and 0.883 for the six simulated test cases.

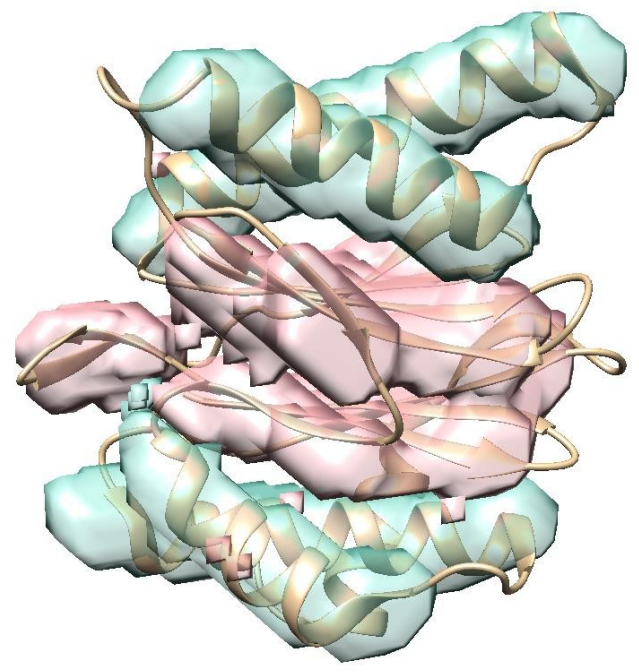

Figure 4. Detected helices and $\beta$-sheets from an experimentally-derived cryo-EM density map 1740 (EMDB ID). The corresponding atomic structure of protein 3c92(PDB ID) (ribbon) is superimposed.

Due to large amount of noise found in experimentally-derived cryo-EM density maps, it is much more challenging to identify secondary structures in such images. An example of segmented helices and $\beta$-sheets is shown for cryo-EM density map EMD-1740 with $6.2 \AA$ resolution (Figure 4). A chain of the protein 3c92 (PDB ID) was used as an envelope to extract the density region that corresponds to the chain in EMD-1740. This chain consists of five helices and three $\beta$-sheets, all of which appear to be segmented correctly (Figure 4). In this case, the average F1 score for 14 patches is 0.819 for helix detection, and 0.853 for $\beta$-sheet detection. The accuracy for cryo-EM case is comparable, with an overall F1 score of 0.828 , to the accuracy of the simulated cases. We plan to expand the amount of training data in future and develop a standard dataset for training. With a larger amount of training data, the model is likely to be more accurate. 
Table 1. Detection accuracy of three classes (helix, $\beta$-sheet, and background). Row 2 to row 7 are simulated test cases using atomic structures of PDB. Row 8 involves an experimentally-derived test case with its EMDB ID indicated in parentheses.

\begin{tabular}{|c|c|c|c|c|c|c|c|}
\hline PDB ID & Patch Number & Helix Number Strand Number & F1-Helix & F1-Sheet & F1-Background & F1-Avg \\
\hline 3j7i_a & 9 & 17 & 9 & 0.806 & 0.749 & 0.812 & 0.789 \\
\hline 1T79 & 10 & 12 & 4 & 0.872 & 0.766 & 0.861 & 0.883 \\
\hline 1cv1 & 8 & 7 & 3 & 0.734 & 0.878 & 0.774 & 0.795 \\
\hline $\mathbf{2 X S 1}$ & 8 & 26 & 2 & 0.81 & 0.998 & 0.802 & 0.87 \\
\hline 3MK4 & 7 & 15 & 2 & 0.8 & 0.999 & 0.794 & 0.864 \\
\hline 4P1T & 7 & 25 & 2 & 0.822 & 0.998 & 0.812 & 0.877 \\
\hline 3C92 (1740) & 14 & 182 & 307 & 0.819 & 0.853 & 0.828 & 0.833 \\
\hline
\end{tabular}

\section{CONCLUSIONS}

Deriving atomic structures from medium-resolution cryo-EM density maps is challenging. An important step to derive the atomic structure automatically is detecting the location of secondary structures within the density map. We have presented a 3D convolutional neural network for segmentation of secondary structure elements from cryo-EM images. Although CNN has been shown as a powerful image processing method, there is limited work developing $\mathrm{CNN}$ architectures that are effective in 3D segmentation problems for protein secondary structure detection from cryo-EM density maps. Using 3D UNET as a guide [32], we have created an encoder decoder architecture employing $3 \mathrm{D}$ convolutions to capture features along three dimensions. We show that this version of 3D U-Net can achieve good accuracy in a test of six simulated density maps and one experimentallyderived cryo-EM map. We plan to improve this model and to perform a large-scale test using more cryo-EM density maps.

\section{ACKNOWLEDGMENTS}

The work in this paper is supported by NSF DBI-1356621 and NIH R01-GM062968. DH, TZ, and JH participated in methodology design. DH implemented and tested the method. $\mathrm{DH}$ and $\mathrm{JH}$ wrote the paper. RL provided initial support.

\section{REFERENCES}

[1] Zheng Liu, Fei Guo, Feng Wang, Tian-Cheng Li, and Wen Jiang. 2016 $2.9 \AA$ Resolution Cryo-EM 3-D Reconstruction of Close-packed Virus Particles. Structure (London, England: 1993) 24, 2 (February 2016), 319328. DOI:https://doi.org/10.1016/j.str.2015.12.006

[2] Xiao-chen Bai, Israel S Fernandez, Greg McMullan, and Sjors HW Scheres. 2013. Ribosome structures to near-atomic resolution from thirty thousand cryo-EM particles. eLife 2, (February 2013). DOI:https://doi.org/10.7554/eLife.00461

[3] Kwok-Yan Chan, Leonardo G. Trabuco, Eduard Schreiner, and Klaus Schulten. 2012. Cryo- Electron Microscopy Modeling by the Molecular Dynamics Flexible Fitting Method. Biopolymers 97, 9 (September 2012), 678-686. DOI:https://doi.org/10.1002/bip.22042

[4] Gunnar F. Schröder, Axel T. Brunger, and Michael Levitt. 2007. Combining Efficient Conformational Sampling with a Deformable Elastic Network Model Facilitates Structure Refinement at Low Resolution. Structure 15, 12 (December 2007), 1630-1641. DOI:https://doi.org/10.1016/j.str.2007.09.021
[5] Willy Wriggers and Stefan Birmanns. 2001. Using Situs for Flexible and Rigid-Body Fitting of Multiresolution Single-Molecule Data. Journal of Structural Biology 133, 2-3 (February 2001), 193-202. DOI:https://doi.org/10.1006/jsbi.2000.4350

[6] Kamal Al Nasr, Lin Chen, Dong Si, Desh Ranjan, Mohammad Zubair, and Jing He. 2012. Building the initial chain of the proteins through de novo modeling of the cryo-electron microscopy volume data at the medium resolutions. In Proceedings of the ACM Conference on Bioinformatics. 490-497. DOI:https://doi.org/10.1145/2382936.2382999

[7] Kamal Al Nasr, Desh Ranjan, Mohammad Zubair, Lin Chen, and Jing He. 2014. Solving the Secondary Structure Matching Problem in CryoEM De Novo Modeling Using a Constrained \$K\$- Shortest Path Graph Algorithm. IEEE/ACM Transactions on Computational Biology and

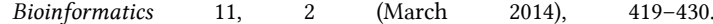
DOI:https://doi.org/10.1109/TCBB.2014.2302803

[8] Kamal Al Nasr, Desh Ranjan, Mohammad Zubair, and Jing He. 2011 Ranking valid topologies of the secondary structure elements using a constraint graph. J Bioinform Comput Biol 9, 3 (June 2011), 415-430.

[9] Devin Haslam, Mohammad Zubair, Desh Ranjan, Abhishek Biswas, and Jing He. 2016. Challenges in matching secondary structures in cryo-EM: An exploration. In Proceeedings of the IEEE International Conference on Bioinformatics and Biomedicine. 1714-1719. DOI:https://doi.org/10.1109/BIBM.2016.7822776

[10] Sasakthi Abeysinghe, Tao Ju, Matthew Baker, Wah Chiu.. 2008. Shape Modeling and Matching in Identifying 3D Protein Structures. ComputerAided Design 40, 6 (June 2008), 708-720. DOI:https://doi.org/ 10.1016/j.cad.2008.01.013.

[11] Matthew L. Baker, Tao Ju, and Wah Chiu. 2007. Identification of Secondary Structure Elements in Intermediate-Resolution Density Maps. Structure 15, 1 (January 2007), 7-19. DOI:https://doi.org/10.1016/j.str.2006.11.008

[12] A. Dal Palù, J. He, E. Pontelli, and Y. Lu. 2006. Identification of alphahelices from low resolution protein density maps. Comput Syst Bioinformatics Conf (2006), 89-98.

[13] Wen Jiang, Matthew L. Baker, Steven J. Ludtke, and Wah Chiu. 2001 Bridging the information gap: computational tools for intermediate resolution structure interpretation. Fournal of Molecular Biology 308, 5 (May 2001), 1033-1044. DOI:https://doi.org/10.1006/jmbi.2001.4633

[14] Yifei Kong and Jianpeng Ma. 2003. A Structural-informatics Approach for Mining $\beta$-Sheets: Locating Sheets in Intermediate-resolution Density Maps. Fournal of Molecular Biology 332, 2 (September 2003), 399-413. DOI:https://doi.org/10.1016/S0022-2836(03)00859-3

[15] Mirabela Rusu and Willy Wriggers. 2012. Evolutionary bidirectional expansion for the tracing of alpha helices in cryo-electron microscopy reconstructions. Fournal of Structural Biology 177, 2 (February 2012), 410-419. DOI:https://doi.org/10.1016/j.jsb.2011.11.029

[16] Dong Si and Jing He. 2013. Beta-sheet Detection and Representation from Medium Resolution Cryo-EM Density Maps. In Proceedings of the International Conference on Bioinformatics, Computational Biology and Biomedical Informatics. 764-770. DOI:https://doi.org/10.1145/2506583.2506707

[17] Zeyun Yu and Chandrajit Bajaj. 2008. Computational Approaches for Automatic Structural Analysis of Large Biomolecular Complexes. IEEE/ACM Transactions on Computational Biology and Bioinformatics 5 , 4 (October 2008), 568-582. DOI:https://doi.org/10.1109/TCBB.2007.70226 
[18] Dong Si and Jing He. 2017. Modeling Beta-Traces for Beta-Barrels from Cryo-EM Density Maps. BioMed Research International 2017, (2017), 1 9. DOI:https://doi.org/10.1155/2017/1793213

[19] Dong Si and Jing He. 2014.Tracing beta strands using StrandTwister from cryo-EM density maps at medium resolutions. Structure 22,11 1665-76.

[20] Lingyu Ma, M. Reisert, and H. Burkhardt. 2012. RENNSH: A Nove alpha-Helix Identification Approach for Intermediate Resolution Electron Density Maps. IEEE/ACM Transactions on Computational Biology and Bioinformatics 9, 1 (January 2012), 228-239. DOI:https://doi.org/10.1109/TCBB.2011.52

[21] Dong Si, Shuiwang Ji, Kamal Al Nasr, and Jing He. 2012. A Machine Learning Approach for the Identification of Protein Secondary Structure Elements from Electron Cryo-Microscopy Density Maps. Biopolymers $\quad 97, \quad 9 \quad$ (September 2012), 698-708. DOI:https://doi.org/10.1002/bip.22063

[22] Rongjian Li, Dong Si, Tao Zeng, Shuiwang Ji, and Jing He. 2016. Deep convolutional neural networks for detecting secondary structures in protein density maps from cryo-electron microscopy. In Proceeedings of the IEEE International Conference on Bioinformatics and Biomedicine. 41-46. DOI:https://doi.org/10.1109/BIBM.2016.7822490

[23] Shuiwang Ji, Wei Xu, Ming Yang, and Kai Yu. 2013. 3D Convolutional Neural Networks for Human Action Recognition. IEEE Transactions on Pattern Analysis and Machine Intelligence 35, 1 (January 2013), 221-231. DOI:https://doi.org/10.1109/TPAMI.2012.59

[24] Alex Krizhevsky, Ilya Sutskever, and Geoffrey E. Hinton. 2017. ImageNet classification with deep convolutional neural networks. Communications of the ACM 60, 6 (May 2017), 84-90. DOI:https://doi.org/10.1145/3065386

[25] Tao Zeng, Rongjian Li, Ravi Mukkamala, Jieping Ye, and Shuiwang Ji. 2015. Deep convolutional neural networks for annotating gene expression patterns in the mouse brain. BMC Bioinformatics 16, 1 (December 2015). DOI:https://doi.org/10.1186/s12859-015-0553-9

[26] Wenlu Zhang, Rongjian Li, Houtao Deng, Li Wang, Weili Lin, Shuiwang Ji, and Dinggang Shen. 2015. Deep convolutional neural networks for multi-modality isointense infant brain image segmentation. NeuroImage

(March 2015) 214-224.
[27]

Dan C. Cireşan, Alessandro Giusti, Luca M. Gambardella, and Jürgen Schmidhuber. 2013. Mitosis Detection in Breast Cancer Histology Images with Deep Neural Networks. In Medical Image Computing and Computer-Assisted Intervention - MICCAI 2013, Kensaku Mori, Ichiro Sakuma, Yoshinobu Sato, Christian Barillot and Nassir Navab (eds.). Springer Berlin Heidelberg, Berlin, Heidelberg, 411-418. DOI:https://doi.org/10.1007/978-3-642-40763-5 51

[28] Y. LeCun, Fu Jie Huang, and L. Bottou. 2004. Learning methods for generic object recognition with invariance to pose and lighting. In Proceedings of the 2004 IEEE Computer Society Conference on Computer Vision and Pattern Recognition. 97-104. DOI:https://doi.org/10.1109/CVPR.2004.1315150

[29] Viren Jain and Sebastian Seung. 2009. Natural Image Denoising with Convolutional Networks. In Advances in Neural Information Processing Systems 21, D. Koller, D. Schuurmans, Y. Bengio and L. Bottou (eds.) Curran Associates, Inc., 769-776. Retrieved from http://papers.nips.cc/paper/3506- natural-image-denoising-withconvolutional-networks.pdf

[30] Srinivas C. Turaga, Joseph F. Murray, Viren Jain, Fabian Roth, Moritz Helmstaedter, Kevin Briggman, Winfried Denk, and H. Sebastian Seung. 2010. Convolutional Networks Can Learn to Generate Affinity Graphs for Image Segmentation. Neural Computation 22, 2 (February 2010), 511-538. DOI:https://doi.org/10.1162/neco.2009.10-08-881

[31] Tao Zeng, Rongjian Li, Ravi Mukkamala, Jieping Ye, and Shuiwang Ji. 2015. Deep convolutional neural networks for annotating gen expression patterns in the mouse brain. BMC Bioinformatics 16, (December 2015). DOI:https://doi.org/10.1186/s12859-015-0553-9

[32] Özgün Çiçek, Ahmed Abdulkadir, Soeren S. Lienkamp, Thomas Brox, and Olaf Ronneberger. 2016. 3D U-Net: Learning Dense Volumetric Segmentation from Sparse Annotation. CoRR. (October 2016), 424-432. https://doi.org/10.1007/978-3-319-46723-8_49

[33] Eric F. Pettersen, Thomas D. Goddard, Conrad C. Huang, Gregory S. Couch, Daniel M. Greenblatt, Elaine C. Meng, and Thomas E. Ferrin 2004. UCSF Chimera?A visualization system for exploratory research and analysis. Fournal of Computational Chemistry 25, 13 (October 2004), 1605-1612. DOI:https://doi.org/10.1002/jcc.20084 